ACCEPTED MANUSCRIPT

\title{
2D bending (curvature) recognition based on combination of a TFBG and an orthogonal TFBG pair
}

To cite this article before publication: Weijia Bao et al 2019 Appl. Phys. Express in press https://doi.org/10.7567/1882-0786/ab2929

\section{Manuscript version: Accepted Manuscript}

Accepted Manuscript is "the version of the article accepted for publication including all changes made as a result of the peer review process, and which may also include the addition to the article by IOP Publishing of a header, an article ID, a cover sheet and/or an 'Accepted

Manuscript' watermark, but excluding any other editing, typesetting or other changes made by IOP Publishing and/or its licensors"

This Accepted Manuscript is @ 2019 The Japan Society of Applied Physics.

During the embargo period (the 12 month period from the publication of the Version of Record of this article), the Accepted Manuscript is fully protected by copyright and cannot be reused or reposted elsewhere.

As the Version of Record of this article is going to be / has been published on a subscription basis, this Accepted Manuscript is available for reuse under a CC BY-NC-ND 3.0 licence after the 12 month embargo period.

After the embargo period, everyone is permitted to use copy and redistribute this article for non-commercial purposes only, provided that they adhere to all the terms of the licence https://creativecommons.org/licences/by-nc-nd/3.0

Although reasonable endeavours have been taken to obtain all necessary permissions from third parties to include their copyrighted content within this article, their full citation and copyright line may not be present in this Accepted Manuscript version. Before using any content from this article, please refer to the Version of Record on IOPscience once published for full citation and copyright details, as permissions will likely be required. All third party content is fully copyright protected, unless specifically stated otherwise in the figure caption in the Version of Record.

View the article online for updates and enhancements. 


\title{
2D bending (curvature) recognition based on combination of a TFBG and an orthogonal TFBG pair
}

\author{
Weijia Bao ${ }^{1,2, *}$, Changle Wang ${ }^{2}$, Yiping Wang ${ }^{1}$, Namita Sahoo ${ }^{2}$, Lin Zhang ${ }^{2}$ \\ 1. Key Laboratory of Optoelectronic Devices and Systems of Ministry of \\ Education and Guangdong Province, College of Optoelectronic Engineering, \\ Shenzhen University, Shenzhen 518060, China
}

2. Aston Institute of Photonic Technologies, Aston University, Birmingham,

B4 7ET, UK

E-mail: wjbao1991@gmail.com

We demonstrated a highly-efficient 2D bending sensor with great direction recognition based on the integration of a titled fiber Bragg grating (TFBG) and a specially-designed orthogonal TFBG pair. The low-order cladding modes of the sensor present significant responses to fiber bending magnitude and direction, which is the key to bending direction recognition. Meanwhile, the stable fundamental core mode can be used to monitor the system power and eliminate the temperature effect. 
Fiber-optic bending (curvature) sensors have attracted considerable interests as their applications in various fields, such as mechanical engineering systems and structural health monitoring of buildings. Generally, the fiber Bragg grating $(\mathrm{FBG})^{1,2)}$ and long period grating $(\mathrm{LPG})^{3,4)}$ based devices can only measure the bending amplitude but not the direction, resulting in some limitations for applications. It has been reported that the fiber interferometers assembled with asymmetric structures ${ }^{5-7)}$ can realize simultaneous measurements of both bending amplitude and direction, but they have intrinsic disadvantages such as complex fabrications, cross-sensitivity and instability, By comparison, special FBG based devices present better performance in directional curvature measurement, attributed to their unique geometric configuration and mode coupling. For instance, the eccentric inscription offers an appropriate mechanism for improving the direction-dependence of $\mathrm{FBG}^{8,9)}$. The grating fabricated in multi-core fiber can also introduce the direction responsivity of FBG devices ${ }^{10-12}$. However the wavelength interrogation of above sensors limits their applicability. Alternatively, the cladding modes excited by the TFBG show high intensity-sensitivity to bending and TFBGs have been demonstrated for orientation-dependent bending measurements ${ }^{13-15)}$. Combining the TFBGs with designed coupling configuration can effectively enhance the cladding mode resonance and further improve sensing performance ${ }^{16-18)}$. Besides, the TFBGs inscribed in specialty fibers also present some directionality and sensitivity improvements ${ }^{19}$.

For a TFBG, the propagation light in fiber core can be transferred into the cladding via titled grating structure, and then multi cladding mode transmission resonances ${ }^{6,8)}$ are excited. Fiber bending can induce an intensity modulation on low-order cladding modes, making the TFBG ideal as a power-reference device. The inherent asymmetric configuration of TFBG is in direct relation to its orientation-dependent characteristic, the tilted grating plane breaks the cylindrical symmetry of the fiber and excites polarization induced pairs of cladding mode resonances which can response differently to fiber bending direction. When a fiber is bent, the core-to-cladding mode coupling efficiency of TFBG is subjected to the angle difference between the bend direction and the grating azimuth plane. However, the orientation-dependent property provided by TFBG cannot distinguish the direction of bending applied in real time. And it requires a dynamic measurement for direction-response recovery ${ }^{8,20)}$, which greatly reduces the measurement efficiency and applicability.

We have proposed and experimentally demonstrated combinational TFBGs based 2D directional bending (curvature) sensing method. Such a sensing configuration contains a normal TFBG and a pair of orthogonal TFBGs (OTFBG pair), of which OTFBG pair is 
assembled by splicing two identical short TFBGs in orthogonal orientation. Due to the bending-direction responses of two orthogonal TFBGs are mutually complementary, the OTFBG pair eliminates the direction dependence, i.e., the OTFBG pair has constant sensitivity for arbitrary bending direction, whereas the normal TFBG presents direction-dependent response to the bending. Therefore, we utilize the OTFBG pair as measuring component of bending magnitude and the normal TFBG as bending-direction reference, and determine the bending direction via mapping in their intensity responses.

We used TFBGs with a tilt angle of $4^{\circ}$ which were fabricated by the phase mask scanning method using an argon ion frequency-doubled $244 \mathrm{~nm}$ laser. The inscribed TFBGs were annealed in a hot oven at $80{ }^{\circ} \mathrm{C}$ for about $48 \mathrm{~h}$ to stabilize the grating structure. The bending measurement system of combinational TFBGs is illustrated in Fig. 1. In the experiment, sensor 1 was placed close to sensor 2 in order to eliminate the difference of applied bending between them. The light from an amplified spontaneous emission (ASE) source was launched in the sensors through a 1x2 coupler, and then their transmission spectral evolution was monitored by an optical spectrum analyzer (OSA). Both sides of these two sensors were fixed on the rotators; the right-side rotator was mounted on a horizontal translation stage. Thus, the vertical bending (opposite to y-direction) applied on the sensors was realized via shifting the translation stage. The bending magnitude is represented by the curvature of bent fiber. The bent part is usually approximated as an arc of circle, so the generated curvature $\mathrm{C}$ can be written as ${ }^{5}$ )

$$
C=\frac{2 d}{d^{2}+L^{2}}
$$

where $L$ is the half length of fiber between the two mounted points, $C$ is the applied curvature, and $d$ is the central displacement of sensor.

The configuration of sensor 1 (normal TFBG) is shown in Fig. 1(b), the grating plane has a certain tilt angle relativel to the fiber center axis (z direction) in the $y$-z plane, and is coincident with the $\mathrm{y}$ axis in the fiber cross section ( $\mathrm{x}-\mathrm{y}$ plane). The transmission spectra of TFBG when applied with several curvatures $\left(0-4 \mathrm{~m}^{-1}\right)$ are shown in Fig. 2(a). The bending-induced strain response of core is negligible beacuse it is located on the neutral axis of fiber and the core can confine the propagation light well, therefore, the high-intensity resonant transmission dip of fundamental core mode remains stable while the applied bending continuously changes, as clearly shown in Fig. 2(a). The core mode is an ideal reference for monitoring system power and temperature fluctuation. The comb-like resonances at further shorter-wavelength side correspond to individual cladding 
mode resonances, which are excited by the strong coupling from the forward-core mode to the backward-cladding modes that is induced by the weakly tilted grating structure. Among them, the lower-order cladding modes are easily recoupled to fiber core by fiber-bending because their mode field powers are mainly distributed in the interface between core and cladding. Thus these lower-order cladding modes are sensitive to fiber-bending. In particular, a "ghost" mode is located on the short-wavelength side of core mode which is a group of cladding modes. Its spectral responses to fiber bending are quite complex and not suitable for bending measurement. And the rest of lower-order cladding modes present the clear measurable intensity variations that can be observed under increasing bending magnitude, as shown in inset of Fig. 2(a).

The dominate contribution to these spectral changes as shown in insert of Fig. 2 is that fiber bending causes non-uniform effective refractive-index variation on the cross-section of fiber due to the photoelastic effect ${ }^{4)}$ and the lateral shift of cladding mode field toward the external fiber portion ${ }^{8}$. Then this lateral shift can decrease the core-to-cladding mode coupling, resulting in cladding mode resonances intensity/decrease in transmission spectrum. As described in $^{15,18)}$, the effective refractive-index variation of a bent TFBG can be defined as

$$
\Delta n=-\left(\frac{n^{3}}{2}\right)\left(p_{12}-v p_{12}-v p_{11}\right) \operatorname{Cr}(\cos \theta \cos \varphi+\sin \theta \sin \varphi)
$$

where $p_{i j}$ is the component of elasto optical tensor with the typical value of $p_{11}=0.12$, $p_{12}=0.27$ for silica, $v$ is the Poisson radio with the value of 0.16 for silica, $r$ is the distance from fiber center, $\theta$ is the azimuth of fiber cross section, and $\varphi$ is the bending direction. According to eq.2, we can see that the effective refractive-index variation is dependent on both fiber bending magnitude $C$ and direction $\varphi$, and $\Delta n$ is determined by the direction $\varphi$ in a certain position within fiber. Since modified effective refractive-index depends on the mode order and polarization components, the coupling coefficient and mode field shifting change differently for each cladding mode. Thus asymmetrically core-to-cladding mode coupling provides strong polarization (orientation) dependence for fiber bending. The direction-dependent bending measurement is then feasible.

We selected the cladding mode at the wavelength of $1548.28 \mathrm{~nm}$ as the monitoring point. With applying the same curvature measurement range from 0 to $4 \mathrm{~m}^{-1}$, TFBG presents various sensitivities versus different directions (see Fig. 2(b)). And for each direction, the intensity change of the cladding mode demonstrates a good linearity. There are also small resonance wavelength variation associated with bending (see insert of Fig. 2(a)), but 
intensity based measuring is easier and more efficient, thus such small wavelength shift can be ignored within defined resolution range. The sensitivity distribution for a full range of $0^{\circ}-360^{\circ}$ bending measurement is shown in Fig. 3, where a strong angular (oriented) dependence of the $2 \mathrm{D}$ bending response is obtained. It shows a variation trend like sine wave. Based on its mechanism and experimental results of directional bending, the intensity response change of TFBG is determined by both bending magnitude and direction for each single 2D bending occurrence. Since the bending sensitivity changes periodically, there is no one-to-one relationship between the bend amplitude and direction, i.e. one bending sensitivity is related to multiple angles for arbitrary bending. So the 2D information cannot be analyzed in single time but can be recovered through multi dynamic measurements with pre calibration. This is fairly low-efficient and not suitable for applications that require real-time monitoring.

To improve the direction discriminating capability, the sensor 2 (OTFBG pair) was introduced for extracting the bending direction information from the measured result of TFBG based bending sensor, which will respond only to the bending magnitude. The response of TFBG to bending involves two kinds of information (bending magnitude and direction). The direction information can be extracted on the basis of the relationship between the intensity change and direction if the magnitude is determined. So we propose a direction-independent sensing component for measuring the bending magnitude and further assisting to identify 2D bending (curvature) direction.

As the above description of direction-dependence of TFBG, the responsivity distribution is in conformity with trigonometric function. In some case the sensitivity will be high and in some cases it will be low, which is dependent on the angular difference between the bending direction and the azimuth of grating plane. Therefore, two sections of TFBG are positioned perpendicularly to each other; their angular difference between the bending direction and the azimuth is $180^{\circ}$. Based on the above analysis, as the schematic diagrams of orthogonal positioned TFBG (OTFBG pair) shown in Fig. 1(c), the responsivity of upstream section 1 and downstream section 2 are complementary. OTFBG pair can predictably achieve a suitable superposition of direction responses and provide a constant direction responsivity, which can effectively eliminate the bending-direction dependence.

The OTFBG pair was simply fabricated by the following method: selecting an identical TFBG with titled angle of $4^{\circ}$ and length of $10 \mathrm{~mm}$, cutting down in the middle of grating and then spliced these two subsections orthogonally to each other as shown in Fig. 1(c). The transmission spectra of the original TFBG and OTFBG pair are shown in Fig. 4(a), the 
fringe visibility of the OTFBG pair is slightly degenerated due to that the orthogonal-spliced process introduce some fusion loss effect on core-to-cladding mode coupling. Adding the OTFBG device into the bending measuring system, the low-order cladding modes also show distinct intensity responses and the core mode keeps unchanged with increasing bending applied, see Fig. 4(b). Unlike the similarly response with TFBG, the OTFBG pair is no longer direction-dependent due to the complementation of dual sensing sections of OTFBG pair. We measured the direction responsivity of OTFBG pair, the sensitivities remain fairly consistent as we expected. From the experimental results shown in Fig.5, we still selected cladding mode around the wavelength around 1548.28nm as the monitoring point, the sensitivity differences were less than $0.03 \mathrm{~dB} / \mathrm{m}^{-1}$ at each bending directions. The overall sensitivity distribution is plotted in Fig. 6, these sensitivities are very close. And it should be noted that the distribution shown in Fig. 6 is still an inconspicuous sine trajectory. It can be caused by the length discrepancy between TFBG of section1 and TFBG of section2, and the coupling efficiency variation induced by imperfect cylindrically symmetrical heating along the/splicing cross section. However, the sensitivity fluctuation is acceptable and sufficient for most applications.

We now perform the 2D directional bending (curvature) measurement. We plot a typical set of results (bending direction range of $0-180^{\circ}$ ) for TFBG and OTFBG pair (for more clearly description, the sensitivity curves are plotted as straight lines). Basing on the proposed sensing system shown in Fig. 1(a), we applied a certain bending from unknown direction, both of OTFBG pair and TFBG provided corresponding intensity responses of $\Delta I_{1}$ and $\Delta I_{2}$. We directly measured the bending magnitude (curvature value) according to the response of OTFBG pair $\left(\Delta I_{1}\right)$, as shown in Fig. $7(\mathrm{a})$. And then, we located the bending direction via combining the measured magnitude and the response of TFBG in Fig. 7(b).

In conclusion, we proposed a 2D directional bending sensor based on combination of a TFBG and an OTFBG pair. The sensing mechanism is based on the directional coupling of low-order cladding mode. Taking advantage of the bending measurement results of the TFBG and OTFBG pair simultaneously, the information of 2D bending magnitude and direction can be efficiently extracted. This device provides a novel solution to the $2 \mathrm{D}$ bending direction recognition. 


\section{Acknowledgments}

This work was supported by National Natural Science Foundation of China (NSFC) (61635007, 61425007); Natural Science Foundation of Guangdong Province (2014A030308007); Science and Technology Innovation Commission of Shenzhen (JCYJ20160427104925452, JCYJ20170412105604705); Development and Reform Commission of Shenzhen Municipality Foundation; China Scholarship Council (CSC, 201706970022) and Initiative Postdocs Supporting Program of 2019 (BX20190217).

\section{References}

1) Taghipour, A. Rostami, M. Bahrami, H. Baghban, M. Dolatyari, "Comparative study between lpfg- and fbg-based bending sensors,” Opt. Commun., 312, 99 (2014).

2) W. J. Zhou, Y. Zhou, X. Y. Dong, L. Y. Shao, J. Cheng. J. Albert, "Fiber-optic curvature sensor based on cladding-mode Bragg grating excited by fiber multimode interferometer,” IEEE Photonics J., 4, 1051 (2012).

3) H. J. Patrick, C. Chang, S. T.Vohra, "Long period fibre gratings for structural bend sensing,” Electron. Lett., 34, 1773 (1998).

4) C. C. Chan, K. M.Tan, C. M.Tay, S. C. Tjin, “Simultaneous measurement of curvature and temperature for LPG bending sensor,” Proc Spie, 5590, 105 (2004).

5) L. Y. Zhang, W. G. Zhang, L. Chen, T. Y. Yan, L. Wang, B. Wang, Q. Zhou, “A Fiber Bending Vector Sensor Based on M-Z Interferometer Exploiting Two Hump-Shaped Tapers,” IEEE. Photonic. Tech. L., 27,1240 (2015).

6) B. Q. Jiang, Z. Y. Bai, C. L. Wang, Y. H. Zhao, J. L. Zhao, L. Zhang, K. M. Zhou, “In-line Mach-Zehnder interferometer with D-shaped fiber grating for temperature-discriminated directional curvature measurement,” J Lightwave. Technol., 36, 742 (2018).

7) H. P. Gong, D. N. Wang, M. L Xiong, C. L. Zhao, K. Ni, “Optical fiber hollow ellipsoid for directional bend sensing with a large bending range,” Opt. Mater. Express., 7, 1767 (2017).

8) W. J. Bao, Q. Z. Rong, F. Y. Chen, X. G. Qiao, “All-fiber 3D vector displacement (bending) sensor based on an eccentric FBG,” Opt.Express., 26, 8619 (2018).

9) D. Feng, X. Qiao, and J. Albert, "Off-axis ultraviolet-written fiber Bragg gratings for directional bending measurements," Opt. Lett. 41, 1201 (2016).

10) A. V. Newkirk, J. E. Antonio-Lopez, A. Velazquez-Benitez, J. Albert, R. Amezcua-Correa, 
and A. Schülzgen, "Bending sensor combining multicore fiber with a mode-selective photonic lantern,” Opt. Lett., 40, 5188 (2015).

11) K. Naeem, Y. Chung, and I. Kwon, "Highly Sensitive Two-Dimensional Bending Vector Sensor Using an Elliptic Two-Core PCF,” IEEE Photonics Technol. Lett., 30, 273 (2018).

12) S. Wang, Y. X. Zhang, W. G. Zhang, P.C Geng, T.Y. Yan, L. Chen, Y. P. Li, and W. Hu, “Two-Dimensional Bending Vector Sensor Based on the Multimode-3-Core-Multimode Fiber Structure,” IEEE Photonics Technol. Lett., 30, 822 (2017).

13) T. Guo, F. Liu, B. O. Guan, J. Albert, “Tilted fiber grating mechanical and biochemical sensors,” Opt. Laser. Technol., 78, 19 (2016).

14) L. Y. Shao, L. Y. Xiong, C. K. Chen, A. Laronche, J. Albert, "Directional Bend Sensor Based on Re-Grown Tilted Fiber Bragg Grating,” J Lightwave. Technol., 28, 2681 (2010).

15) J. Albert, L. Y. Shao, C. Caucheteur, “Tilted fiber Bragg grating sensors,” Laser. Photonics. Rev., 7, 83-108 (2013).

16) T. Guo, L. Y. Shao, H. Y. Tam, P. A. Krug, J. Albert, “Tilted fiber grating accelerometer incorporating an abrupt biconical taper for cladding to core recoupling,” Opt.Express., 17, 20651 (2009).

17) L. Y. Shao, A. Laronche, M. Smietana, P. Mikulic, W, J. Bock, J. Albert, "Highly sensitive bend sensor with hybrid long-period and tilted fiber Bragg grating,” Opt. Commun., 283, 2690 (2010).

18) D. Feng, W. Zhou, X. Qiao, and J. Albert, "Compact Optical Fiber 3D Shape Sensor Based on a Pair of Orthogonal Tilted Fiber Bragg Gratings," Sci. Rep. 5, 17415 (2015).

19) T. Guo, L. B. Shang, Y. Ran, B. O. Guan, J. Albert, “Fiber-optic vector vibroscope,” Opt. Lett., 37, 2703 (2012).

20) Z. C. Zhang, T. Guo, X. J. Zhang, J. Xu, W. P. Xie, M. Nie, Q. Wu, B. O Guan, J. Albert, “Plasmonic fiber-optic vector magnetometer,” Appl. Phys. Lett., 108, 101105 (2016). 


\section{Template for APEX (Jan. 2014)}

\section{Figure Captions}

Fig. 1. Schematic diagrams of (a) experimental setup for bending measurement, (b) TFBG and (c) orthogonal TFBG.

Fig. 2. (a) Transmission spectra of the TFBG versus various curvatures, (b) linear responses of the selected cladding mode versus increasing curvature in different dírection, insets show the bending-induced spectral responses of the cladding mode resonances around $1548.28 \mathrm{~nm}$.

Fig. 3. Sensitivities of the selected cladding mode versus bending direction from $0^{\circ}$ to $360^{\circ}$.

Fig. 4. (a) Transmission spectrum contrast of original TFBG and orthogonal-spliced TFBG, (b) transmission spectra of the OTFBG versus various curvatures.

Fig. 5. Linear responses of the selected cladding mode versus increasing curvature in different direction, insets show the bending-induced spectral responses of the cladding mode resonances around $1548.28 \mathrm{~nm}$.

Fig. 6. Sensitivities of the selected cladding mode versus bending direction from $0^{\circ}$ to $360^{\circ}$, insert shows the superposition schematic of two sections of OTFBG.

Fig. 7. The linear response of (c) OTFBG and (d) TFBG. 
Template for APEX (Jan. 2014)

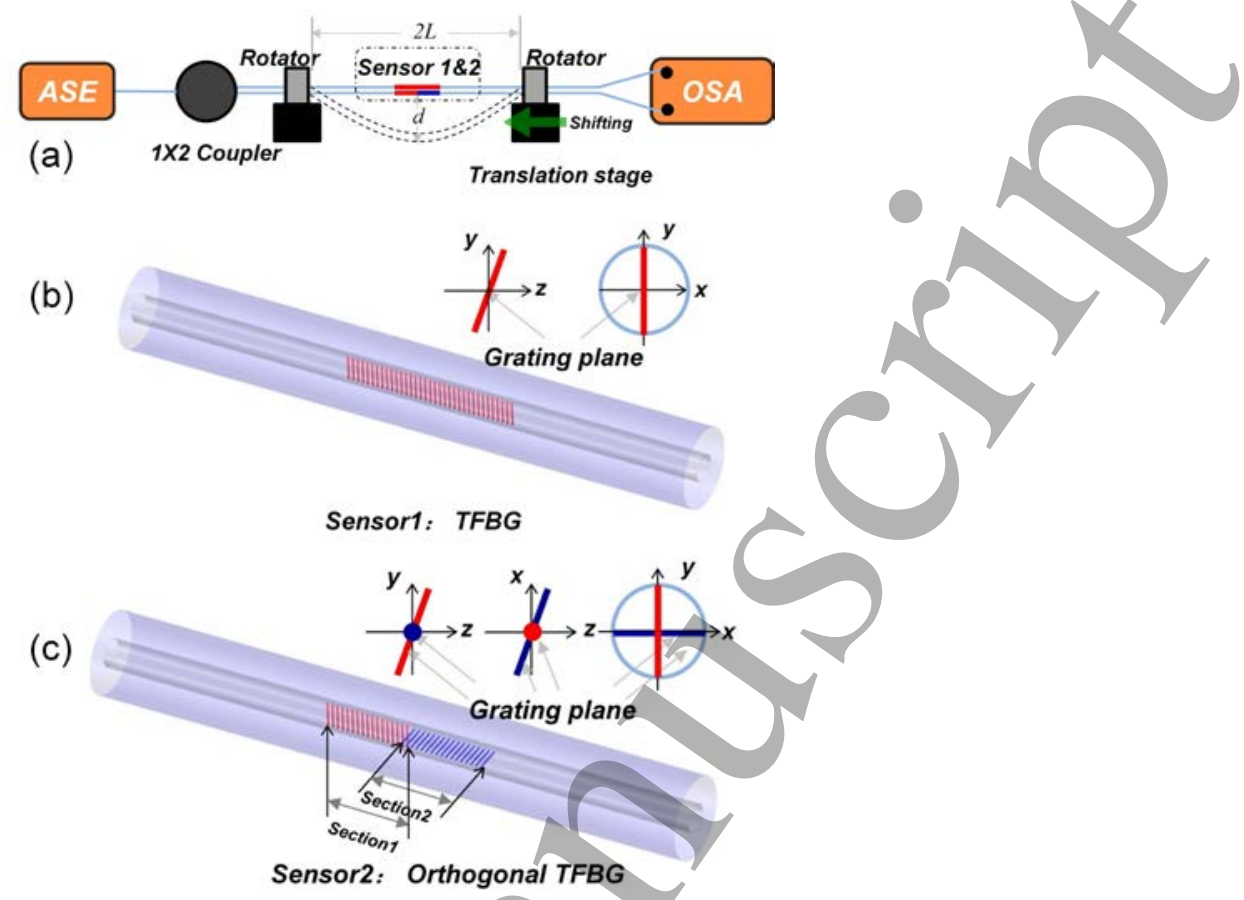

Fig.1.

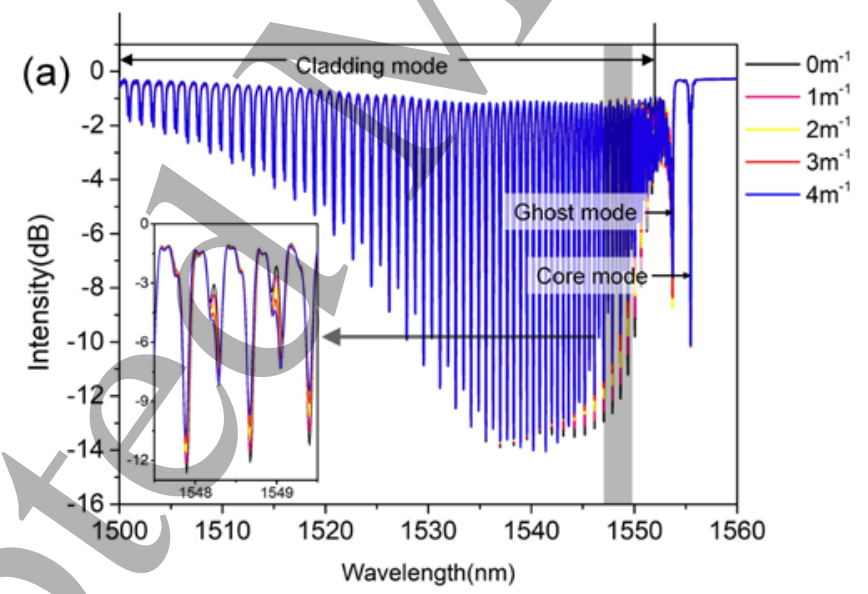




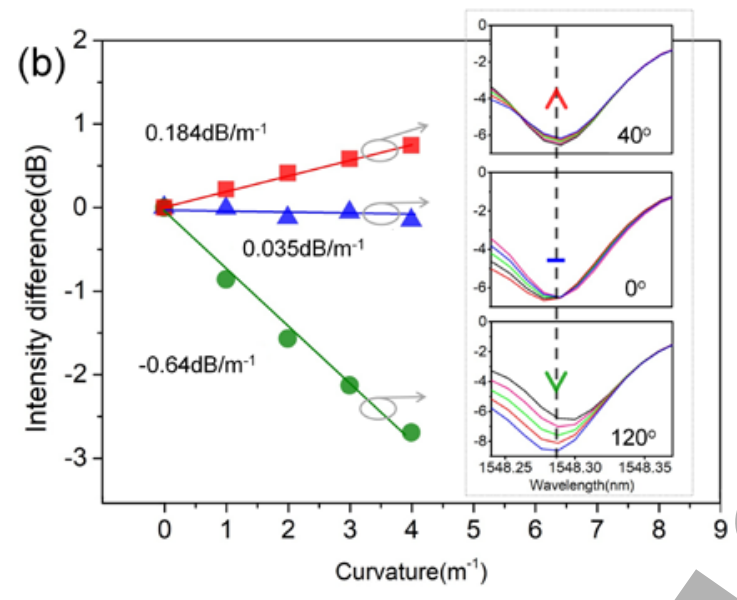

Fig. 2.

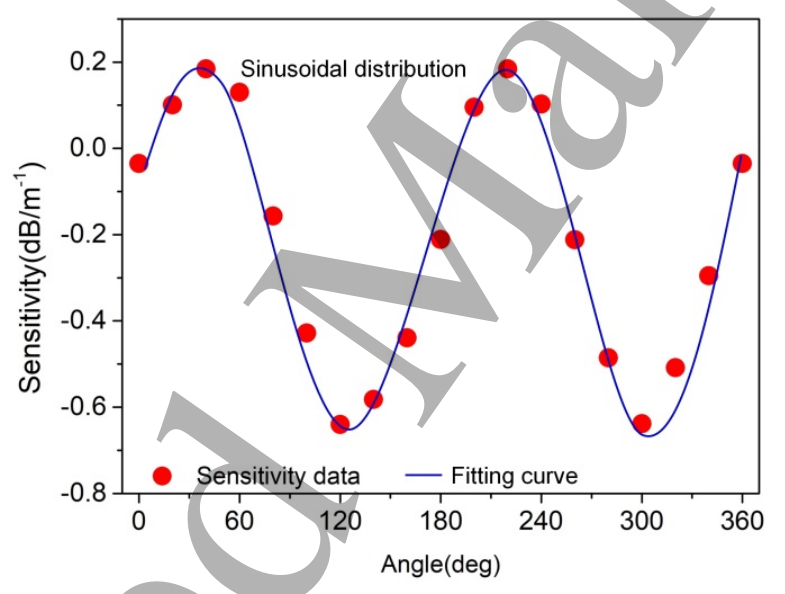

Fig. 3. 

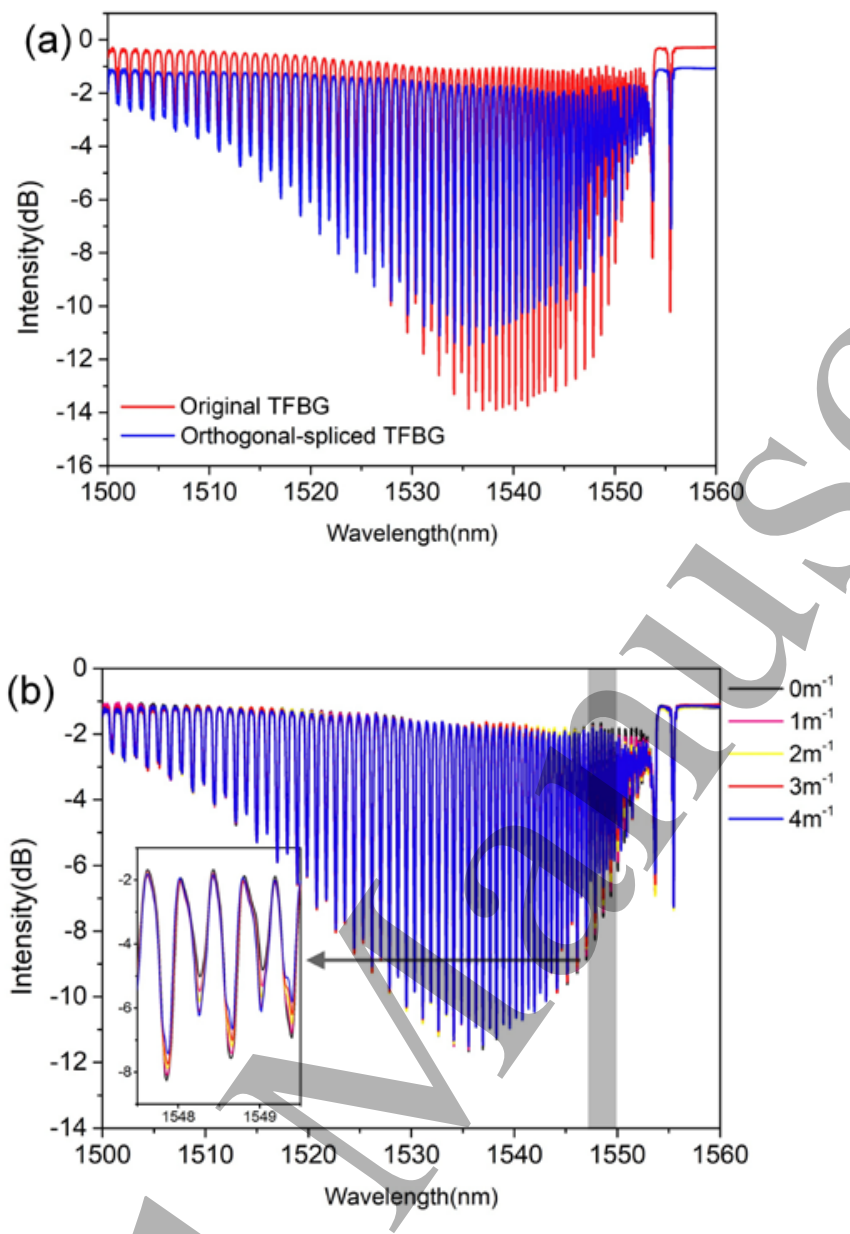

Fig. 4.

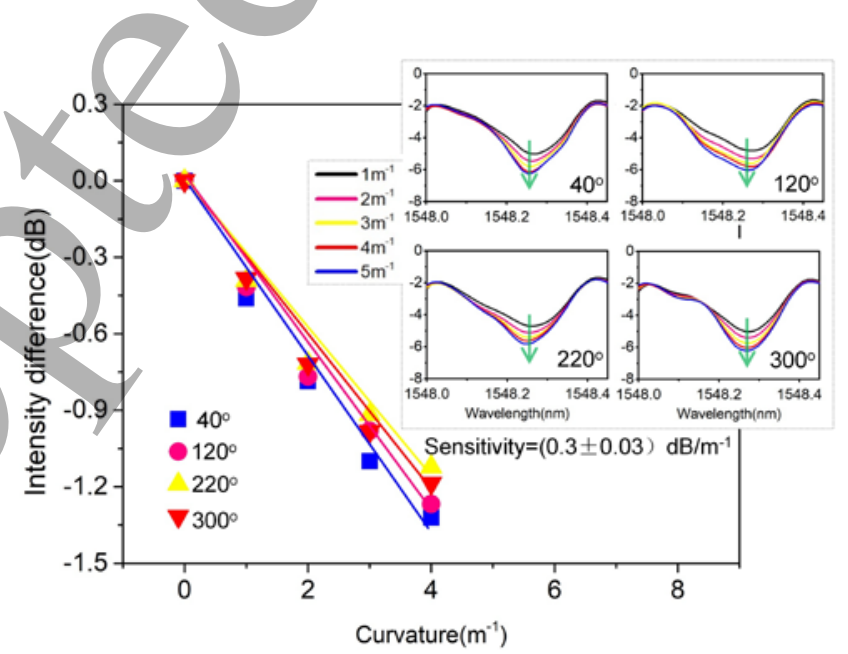

Fig. 5. 


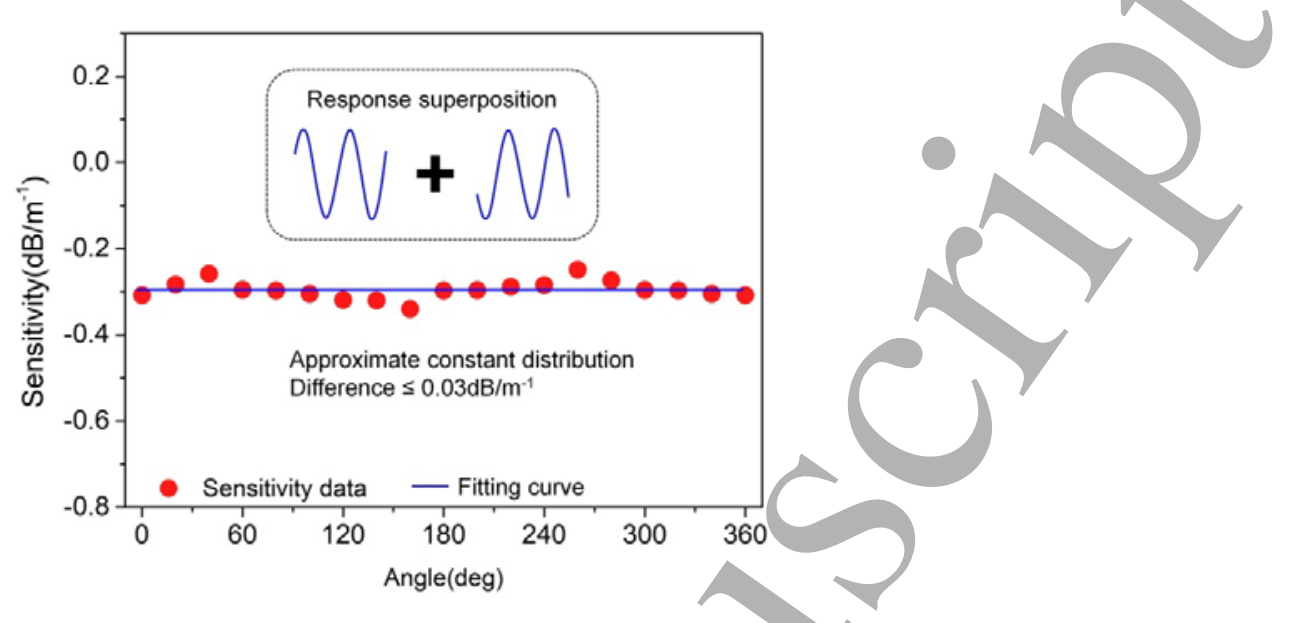

Fig. 6.

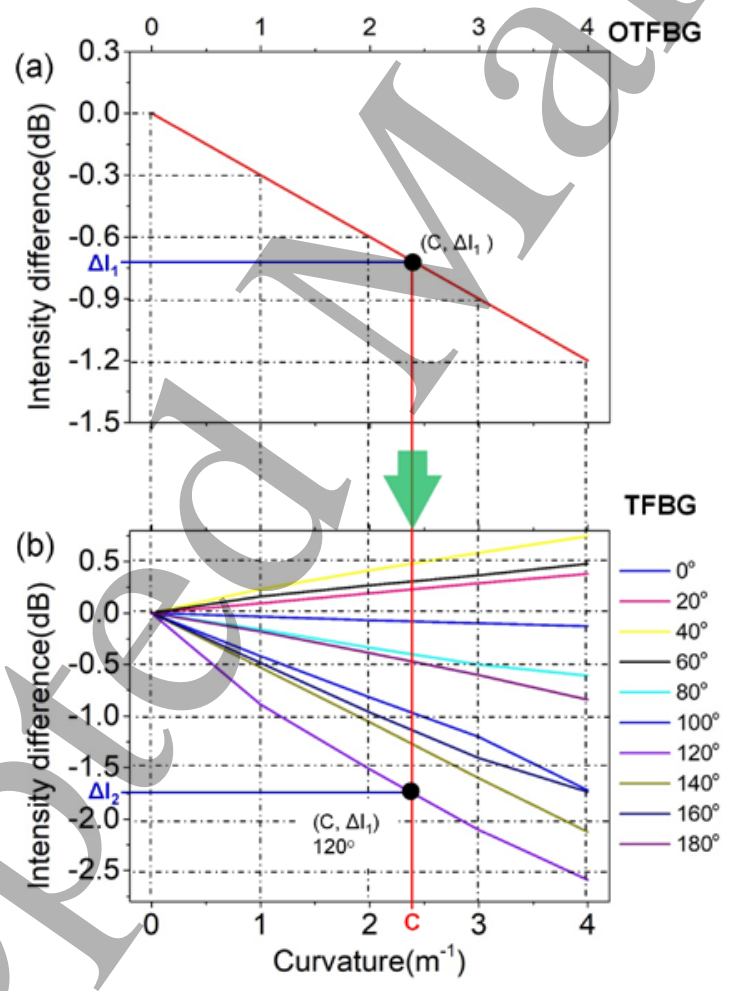

Fig. 7. 